\title{
Effects of Antiretroviral Agents During Pregnancy on Liver Enzymes and Amylase in HIV-Exposed, Uninfected Newborn Infants
}

\author{
Patrícia El Beitune ${ }^{1,2,3}$, Geraldo Duarte ${ }^{1}$, Oona Campbell ${ }^{2}$, Silvana Maria Quintana ${ }^{1}$ and Laura C. Rodrigues ${ }^{2}$ \\ ${ }^{1}$ Department of Obstetrics and Gynecology, Faculty of Medicine of Ribeirão Preto, University of São Paulo; Ribeirão Preto, SP, Brazil; \\ ${ }^{2}$ Infectious Disease Epidemiology Unit, London School of Hygiene \& Tropical Medicine; London, UK; ${ }^{3}$ Department of Obstetrics and \\ Gynecology, Fundação Faculdade Federal de Ciências Médicas de Porto Alegre; Porto Alegre, RS, Brazil
}

\begin{abstract}
This study assessed the effect of antiretroviral drugs administered to pregnant women on amylase and liver enzymes of the neonate. A prospective study was conducted on 52 neonates divided into three groups: infants born to HIVinfected mothers taking zidovudine (ZDV group, $n=18$ ), infants born to mothers taking zidovudine + lamivudine + nelfinavir (TT group, $n=22$ ) and infants born to normal women (control group, $n=12$ ). Umbilical cord blood from the newborn infant was used to determine liver transaminases and amylase. Data were analyzed statistically by nonparametric tests, with the level of significance set at $\mathbf{p}<\mathbf{0 . 0 5}$. The median levels for $\mathrm{TT}$ group newborns were 33.3 $\mathrm{U} / \mathrm{L}$ for oxaloacetic transaminase, $21.5 \mathrm{U} / \mathrm{L}$ for pyruvic transaminase, $1.9 \mathrm{mg} / \mathrm{dL}$ for total bilirubin, $153 \mathrm{mg} / \mathrm{dL}$ for alkaline phosphatase, and 9.6 U/L for amylase. These results did not differ from those obtained for Control newborns or newborns exposed to ZDV alone. No association was observed between the use of antiretroviral drugs during pregnancy and adverse effects on neonatal amylase and hepatic parameters at birth.
\end{abstract}

Key-Words: Combination antiretroviral therapy, hypothesis generation, infant toxicity, umbilical cord blood.

Combination therapy has been increasingly used for the prevention of mother-to-child transmission of HIV because it has been shown to be more effective [1,2]. Nucleoside reverse transcriptase inhibitors cross the placenta, with a maternalfetal drug transfer ratio close to 0.85 for zidovudine (ZDV), 1.0 for lamivudine (3TC), and minimal for protease inhibitors, including nelfinavir (NFV). Although this maternal-fetal transfer is beneficial regarding the prevention of the vertical transmission of HIV-1, antiretroviral agents (ARV) may expose the infant to the risk for adverse effects [2-4].

Elevated transaminase levels are observed in the presence of acute hepatic damage secondary to the exposure to hepatotoxic agents, and serum amylase concentrations may be used to determine, as first analysis, direct toxicity to the acinar cells of the pancreas. Despite a reasonable interest in this evaluation, there have been no studies evaluating prospectively the effects of maternal ARV use on the hepatic parameters and serum amylase of the neonates at birth [3]. Therefore, in the present study we assessed the effects of two different intrauterine exposures, i.e., prophylactic use of $\mathrm{ZDV}$ or triple antiretroviral treatment (ZDV $+3 \mathrm{TC}+\mathrm{NFV})$, on the transaminase, bilirubin and alkaline phosphatase levels and serum amylase levels of HIV-exposed uninfected neonates at birth.

\section{Materials and Methods}

We carried out a prospective cohort study on 57 women aged 16 to 43 years with singleton gestations. Forty-five of

Received on 6 January 2007; revised 19 May 2007.

Address for correspondence: Dr. Patrícia El Beitune. Rua Prof. Annes Dias, 285. Maternidade Mário Totta, $1^{\circ}$ andar. Complexo Hospitalar Santa Casa. Zip code: 90020-090. Phone: 0XX.51.3214.8525. E-mail: pbeitune@fffcmpa.edu.br.

The Brazilian Journal of Infectious Diseases

2007;11(3):314-317 (C) 2007 by The Brazilian Journal of Infectious Diseases and Contexto Publishing. All rights reserved. these women were infected with HIV-1 and the remaining 12 were normal in both clinical and laboratory terms and all subjects were selected at the time when they started prenatal care. The present study was approved by the Research Ethics Committee of the Institution and written informed consent to participate was obtained from each subject.

The HIV-1-infected women were divided into two groups named ZDV group and triple treatment (TT) group. The ZDV group consisted of 20 pregnant women who fulfilled the requirements for the prophylactic use of $\mathrm{ZDV}\left(\mathrm{CD}_{4}>500\right.$ cells/ $\mathrm{mm}^{3}$ and viral load $<1,000$ copies $/ \mathrm{mL}$ ). The TT group consisted of 25 pregnant women with a clinical and laboratory indication $\left(\mathrm{CD}_{4}<500\right.$ cells $\left./ \mathrm{mm}^{3}\right)$ for triple antiretroviral treatment (ZDV + $3 T C+$ NFV) according to the criteria established by the Perinatal HIV Guidelines Working Group Members regarding antiretroviral treatment of pregnant women [4]. HIV-infected patients who had not been treated previously with antiretroviral drugs were selected for the study. The antiretroviral agents recommended since the $14^{\text {th }}$ week were $\mathrm{ZDV}, 300 \mathrm{mg} /$ dose, twice a day for the ZDV group and $300 \mathrm{mg}$ ZDV, $150 \mathrm{mg} 3 \mathrm{TC}$ and $1250 \mathrm{mg}$ NFV in two daily doses for the TT group.

We included for analysis gestational age at birth, alcohol use, illicit drugs use during gestation, and tobacco use. We excluded pregnant women with maternal hypertension, diabetes mellitus, women who did not comply with the use of antiretroviral drugs or used them irregularly, women taking other medications, HIV-infected infants and newborn infants with congenital infections.

Blood was obtained from pregnant women at two different times during gestation (14-20 and 33-38 weeks) for the determination of serum aminotransferases and amylase and umbilical cord blood was obtained immediately after birth for analysis of liver enzymes (alanine aminotransferase (ALT/ SGPT), aspartate aminotransferase (AST/SGOT), bilirubin, alkaline phosphatase and serum amylase). These 
determinations could not be concluded in two ZDV group and three TT Group infants due to concomitance of exclusion criteria. Serum was collected into a vacutainer tube without anticoagulants and the analyses were carried out by an automated method using the Behring Dimension RXL apparatus.

A pilot study was first carried out using the data obtained from 10 newborn infants born to HIV-uninfected mothers and from 10 additional neonates who had an indication for triple antiretroviral agents during the intrauterine period and the mean and standard deviation for the groups were calculated. Since we had hypothesized that use of antiretroviral drugs during pregnancy would cause an elevation of aminotransferases and amylase compared to control, a test was performed to calculate sample size by comparing means by the unpaired one-tailed t-test, with $\alpha=0.05$ and $\beta=20 \%$, indicating the need for a minimum sample of 12 to demonstrate a difference range of 35-40 percent related to aminotransferases and amylase. Conversely, it has been demonstrated the need for a sample of 18 newborn infants for the study groups to demonstrate a difference range of 25-34 percent related to the same variables, respectively. The software GraphPad StatMate 1.01 was used for these calculations.

The variability of liver enzymes (ALT/SGPT, AST,/SGOT) and serum amylase levels was calculated on the basis of the median and interquartile variation (1st and 3rd quartile, respectively). The nonparametric chi-square $\left(\chi^{2}\right)$, MannWhitney and Kruskal-Wallis tests were used, with the level of significance set at $\mathrm{p}<0.05$. All analyses were performed using the SPSS 10.0 software.

\section{Results}

Median gestational age at delivery was 39 weeks for the control group, 38.1 weeks for the ZDV group and 38.5 weeks for the TT group (Kruskal-Wallis, $p=0.57$ ). Median infant weight was 3,250, 3,080 and 3,100 $\mathrm{g}$ for the Control, ZDV and TT groups, respectively (Kruskal-Wallis, $\mathrm{p}=0.45$ ).

The proportion of tobacco consumption was 9.0,40.0 and 20.0 percent for the control, ZDV and TT groups, respectively. No significant difference was observed among groups $\left(\chi^{2}\right.$ test, $\mathrm{p}=0.10)$. Alcohol consumption was reported by 17.0 , 30.0 and 48.0 percent of the control, ZDV and TT pregnant women. These data also did not differ significantly among groups $\left(\chi^{2}\right.$ test, $\left.\mathrm{p}=0.14\right)$. None of the pregnant women reported the use of illicit drugs during pregnancy.

Table 1 presents the results concerning the variables for the pregnant women, i.e., AST, ALT and amylase concentrations, at two times during pregnancy (14-20 and 3338 weeks). Median AST levels at 14-20 weeks were 20.5 U/L for the control group, $24 \mathrm{U} / \mathrm{L}$ for the ZDV group and $28 \mathrm{U} / \mathrm{L}$ for the TT group. No difference was observed among groups (Kruskal-Wallis test, $\mathrm{p}=0.16$ ). At 33-38 weeks, median AST levels were 24.0, 27.5 and 30.0 for the control, ZDV and TT groups, respectively (Kruskal-Wallis test, $\mathrm{p}=0.33$ ). For the TT Group, the $75^{\text {th }}$ percentile was equal to or less than $36.5 \mathrm{U} /$
L for ALT and equal to or less than 48.0 U/L for amylase. No significant difference was observed among groups regarding ALT or amylase levels (Kruskal-Wallis test, $\mathrm{p}=0.12$ and $\mathrm{p}=$ 0.22 , respectively).

Table 2 presents the results concerning the variables for the newborns, i.e., ALT, AST, total bilirubin and amylase concentrations detected in umbilical cord blood from the newborn at the time of delivery. Analysis of these variables demonstrated no significant differences between the newborns of the TT and ZDV groups and the control group (Mann-Whitney test, $\mathrm{p}>0.10$ ).

\section{Discussion}

3TC and ZDV are classified by the Food and Drug Administration (FDA) as category $\mathrm{C}$, indicating there are no controlled studies in women and that animal studies are either not available or have revealed adverse effects. Thus, these drugs should only be given if the potential benefits justify the potential risks to the fetus. 3TC readily crosses the placenta in humans, achieving comparable cord blood and maternal concentrations [5], with pharmacokinetics similar to that observed in non-pregnant adults and with no pharmacokinetic interactions with ZDV [4]. Conversely, ZDV rapidly crosses the human placenta, achieving ratio of cord to maternal blood of about 0.80 . NFV is classified as FDA pregnancy category $\mathrm{B}$, indicating that animal reproduction studies have failed to demonstrate a risk to the fetus, and well-controlled studies of pregnant women have not been conducted. On the other hand, in a phase I study of pregnant women and their infants, transplacental passage of NFV was minimal [4]. In a study of cord blood samples from 38 women who were treated with NFV during pregnancy, the cord blood NFV concentration was below the assay limit of detection in $63 \%$ of samples and was low (median, $0.35 \mathrm{mcg} / \mathrm{mL}$ ) in the remaining women [6]. Another study demonstrated median cord and maternal NFV concentrations lower than 250 and $1110 \mathrm{ng} / \mathrm{mL}$, respectively. It has been suggested that limited transfer of protease inhibitors may result from their high degree of plasma protein binding and their backwards transport through P-glycoprotein, largely expressed in the placenta [7]. Although there have been few studies analyzing adverse metabolic and biochemical effect of maternal ARV drugs on infant health [8], there have been no reports of the hepatic parameters of newborn infants during the immediate postnatal period [3].

ARV drugs are associated with changes in hepatic parameters. It has been previously demonstrated that hepatotoxicity secondary to the use of ARV drugs occurs in $2 \%$ of the patients exposed to highly active ARV therapy [9]. In general, all nucleoside analogues have been cited in these statements, although their hepatotoxic potential differs, being more prevalent for stavudine (d4T), zalcitabine (ddC) and didanosine (ddI) than for abacavir, ZDV and lamivudine, according to their greater interference with mitochondrial DNA synthesis. In addition, protease inhibitors are also associated with hepatic toxicity $[10,11]$. 
Table 1. Aspartate aminotransferase (AST), alanine aminotransferase (ALT) and amylase levels obtained from pregnant women at two different periods during gestation

\begin{tabular}{lcccc}
\hline Variables & Control $(\mathbf{n}=\mathbf{1 2})$ & $\mathbf{Z D V}(\mathbf{n}=\mathbf{2 0})$ & TT $(\mathbf{n}=\mathbf{2 5})$ & $\mathbf{p}^{*}$ \\
\hline AST & & & & \\
14-20 weeks & 20.5 & 24.0 & 28.0 & 0.16 \\
AST & $(16.5-31.5)$ & $(22.0-28.0)$ & $(24.5-30.5)$ & \\
33-38 weeks & 24.0 & 27.5 & 30.0 & 0.33 \\
ALT & $(18.5-32.0)$ & $(24.5-31.5)$ & $(25.5-34.0)$ & \\
14-20 weeks & 17.5 & & & \\
ALT & $(15.5-23.5)$ & $(21.0-26.5)$ & $(16.0-28.0)$ & 0.15 \\
33-38 weeks & 24.0 & 31.0 & 32.0 & \\
Amylase & $(18.0-33.0)$ & $(26.5-33.5)$ & $(28.0-36.5)$ & \\
14-20 weeks & 33.5 & 33.5 & 35.0 & \\
Amylase & $(25.5-35.5)$ & $(30.0-36.5)$ & $(30.5-40.5)$ & 0.12 \\
33-38 weeks & 37.5 & 35.5 & 39.0 & 0.22 \\
& $(30.5-43.0)$ & $(32.0-39.5)$ & $(34.5-48.0)$ & \\
\hline
\end{tabular}

ZDV group, pregnant women taking zidovudine alone. TT group, pregnant women taking zidovudine + lamivudine + nelfinavir. *Kruskal-Wallis test. Data are reported as medians and $1^{\text {st }}$ and $3^{\text {rd }}$ quartiles in U/L.

Table 2. Alkaline phosphatase (AP), total bilirubin (TB), aspartate aminotransferase (AST), alanine aminotransferase (ALT), and amylase levels obtained in umbilical cord blood from newborns at the time of delivery

\begin{tabular}{lcccccccccc}
\hline Group & $\begin{array}{c}\mathbf{A P} \\
\mathbf{m g} / \mathbf{d L}\end{array}$ & $\mathbf{p}$ & $\begin{array}{c}\mathbf{T B} \\
\mathbf{m g} / \mathbf{d L}\end{array}$ & $\mathbf{p}^{*}$ & $\begin{array}{c}\mathbf{A S T} \\
\mathbf{U} / \mathbf{L}\end{array}$ & $\mathbf{p}^{*}$ & $\begin{array}{c}\mathbf{A L T} \\
\mathbf{U} / \mathbf{L}\end{array}$ & $\mathbf{p}^{*}$ & $\begin{array}{c}\text { Amylase } \\
\mathbf{U} / \mathbf{L}\end{array}$ & $\mathbf{p}^{*}$ \\
\hline Control & 134 & -- & 2.0 & -- & 36.7 & -- & 17.6 & -- & 15.8 & \\
$(\mathrm{n}=12)$ & $(97-169)$ & & $(1.8-2.2)$ & & $(31-43)$ & & $(15.7-21)$ & & $(12.9-18.0)$ & -- \\
$\mathrm{ZDV}$ & 133 & 0.31 & 1.8 & 0.58 & 32.2 & 0.79 & 16.9 & 0.81 & 14.3 & 0.58 \\
$(\mathrm{n}=18)$ & $(117-189)$ & & $(1.6-2.5)$ & & $(27-56)$ & & $(12-26)$ & & $(9.7-17.6)$ & \\
$\mathrm{TT}$ & 153 & 0.32 & 1.91 & 0.37 & 33.3 & 0.54 & 21.5 & 0.11 & 9.6 & 0.18 \\
$(\mathrm{n}=22)$ & $(127-174)$ & & $(1.5-2.2)$ & & $(28-53)$ & & $(15.6-31.5)$ & & $(7.0-16.0)$ & \\
\hline
\end{tabular}

*Control group x ZDV and TT groups: p>0.05. Mann-Whitney test. ZDV group, infants born to mother taking zidovudine alone. TT group, infants born to mothers taking zidovudine + lamivudine + nelfinavir. Data are reported as medians and 1st and 3rd quartiles.

None of the pregnant women enrolled in the present study showed hepatotoxicity. Among the ARV agents frequently used during pregnancy, NFV is rarely evoked as an agent directly related to hepatotoxicity. The careful monitoring for hepatotoxicity after initiation of ARV therapy is important since it has been demonstrated that the side effects of ARV therapy are more frequent in pregnant than in non-pregnant women. This situation is more often demonstrated with the use of nevirapine [12,13].

In the present study, the newborns of the control group had median levels of SGOT/AST of 36.7 U/L, median levels of SGPT/ALT of $17.6 \mathrm{U} / \mathrm{L}$, total bilirubin of $2.0 \mathrm{mg} / \mathrm{dL}$, direct bilirubin of $0.53 \mathrm{mg} / \mathrm{dL}$, and alkaline phosphatase of $134 \mathrm{mg} /$ dL. These results did not differ from those obtained from newborns exposed to ZDV alone or to combined ARV schemes during the prenatal period. The present data differ from those reported by Silverman et al. [14], who observed discreet elevation of hepatic function, especially AST concentrations, in 58\% of the newborns evaluated, even though none of them presented symptoms of jaundice. The data obtained in the present study are similar to those obtained in other studies $[15,16]$, permitting us to infer that the use of ARV drugs during pregnancy and delivery, whether in separate or combined ARV schemes such as those used in the present series, does not interfere with the hepatic parameters of the infants at birth.

Analysis of serum amylase concentrations in umbilical cord blood at birth revealed that the newborns of the control group presented a median value of $15.8 \mathrm{U} / \mathrm{L}$, which did not differ significantly from the values observed in the ZDV and TT groups. The interest in the determination of this biochemical parameter, despite its recognized nonspecific nature, is due to the observation that several medications frequently used by 
HIV-infected patients are associated with subclinical pancreatitis, possibly due to direct toxicity to the acinar cells of the pancreas [17]. Among the medications frequently associated with damage to exocrine pancreatic function are didanosine, pentamidine, the sulfonamides, corticosteroids, and zalcitabine. 3TC has been associated with the possible deterioration of pancreatic function in preliminary reports regarding HIV-1-infected children taking this medication, although this result was not confirmed in subsequent studies conducted on adults. According to these investigators, no association was observed between the use of ZDV and protease inhibitors and the elevation in amylase concentration, although this statement was contested by others [18] who observed a tendency to an elevation of this pancreatic enzyme in patients taking ZDV alone.

Although a larger number of pregnant women would have been better for detecting an effect of lower magnitude among groups, at present, this study is the single prospective cohort study that had addressed the effect of combination ARV therapy used throughout gestation on serum transaminase and amylase levels obtained from umbilical blood cord immediately at birth, and has included all eligible women diagnosed with HIV during the study period and their neonates (September 2001 to March 2003). Our results may demonstrate with reasonable assurance that the ARV agents used during pregnancy did not cause an elevation greater than $35 \%-40 \%$ among the infants of the groups studied.

The results obtained from the present series suggest safety of the use of ARV drugs during pregnancy regarding liver enzymes and amylase in HIV-exposed, uninfected newborn infants. While our data are reassuring, we emphasize the need for careful monitoring for hepatotoxicity after initiation of antiretroviral therapy. Follow-up is still limited and needs to be continued into adulthood before it can be concluded that there is no potential toxicity of maternal ARV treatment $[8,19]$.

\section{Acknowledgements}

Research supported by FAPESP (Grant number 01/084508). We thank the Central Laboratory of the University Hospital, Faculty of Medicine of Ribeirão Preto, University of São Paulo, for the laboratory tests and Mrs. Elettra Greene for her technical services.

\section{References}

1. Minkoff H., Ahdieh L., Watts D.H., et al. The relationship of pregnancy to the use of highly active antiretroviral therapy. Am J Obstet Gynecol 2001;184:1221-7.
2. Mandelbrot L., Peytavin G., Firtion G., Farinotti R. Maternalfetal transfer and amniotic fluid accumulation of Lamivudine in human immunodeficiency virus-infected pregnant women. Am J Obstet Gynecol 2001;184:153-8.

3. El Beitune P., Duarte G., Quintana S.M., et al. Antiretroviral Therapy during pregnancy and early neonatal life: consequences for HIVexposed, uninfected children. Braz J Infect Dis 2004;8:140-50.

4. Perinatal HIV Guidelines Working Group Members. Public Health Service Task Force Recommendation for Safety and Toxicity of Individual Antiretroviral Agents in Pregnancy. Available at http://www.hivatis.org, retrieved June 15, 2006.

5. Moodley J., Moodley D., Pillay K., et al. Pharmacokinetics and antiretroviral activity of lamivudine alone or when coadministered with zidovudine in human immunodeficiency virus type 1-infected pregnant women and their offspring. J Infect Dis 1998; 178:1327-33.

6. Mirochnick M., Dorenbaum A., Holland D., et al. Concentrations of protease inhibitors in cord blood after in utero exposure. Pediatr Infect Dis J 2002;21:835-8.

7. Marzolini C., Rudin C., Decosterd L.A., et al. Transplacental passage of protease inhibitors at delivery. AIDS 2002;16:889-93.

8. El Beitune P., Duarte G., Foss M.C., et al. Effect of maternal use of antiretroviral agents on serum insulin levels of the newborn infant. Diabetes Care 2005;28:856-9.

9. Rodriguez-Rosado R., Garcia-Samaniego J., Soriano V. Hepatotoxicity after introduction of highly active antiretroviral therapy. AIDS 1998;12:1256.

10. Dieterich D. Managing antiretroviral-associated liver disease. J Acquir Immune Defic Syndr 2003;34:S34-9.

11. Puoti M., Torti C., Diego R., et al. Severe hepatotoxicity during combination antiretroviral treatment: incidence, liver histology, and outcome. J Acquir Immune Defic Syndr 2003;32:259-67.

12. Hill J.B., Shfeffield J.S., Zeeman G.G., Wendel G.D. Jr. Hepatotoxicity with antiretroviral treatment of pregnant women. Obstet Gynecol 2001;98:909-11.

13. Timmermans S., Tempelman C., Godfried M.H., et al. Nelfinavir and nevirapine side effects during pregnancy. AIDS 2005;19:795-9.

14. Silverman N.S., Watts D.H., Hitti J., et al. Initial multicenter experience with double nucleoside therapy for human immunodeficiency virus infection during pregnancy. Infect Dis Obstet Gynecol 1998;6:237-43.

15. Taha T.E., Kumwenda N., Gibbons A., et al. Effect of HIV-1 antiretroviral prophylaxis on hepatic and hematological parameters of African infants. AIDS 2002;16:851-8.

16. Alimenti A., Burdge D.R., Ogilvie G.S., et al. Lactic acidemia in human immunodeficiency virus-uninfected infants exposed to perinatal antiretroviral therapy. Pediatr Infect Dis J 2003; $22: 782-8$

17. Dassopoulos T., Ehrenpreis E.D. Acute pancreatitis in human immunodeficiency virus-infected patients: a review. Am J Med 1999; $107: 78-84$.

18. Argiris A., Mathur-Wagh U., Wilets I., Mildvan D. Abnormalities of serum amylase and lipase in HIV-positive patients. Am J Gastroenterol 1999;94:1248-52.

19. El Beitune P., Duarte G. Antiretroviral agents during pregnancy: Consequences on hematologic parameters in HIV-exposed, uninfected newborn infant. Eur J Obstet Gynecol Reprod Biol 2006; $128: 59-63$. 